\title{
Estudo comparativo entre imagens SRTM e cartas do exército para estudo plano altimétrico em bacias hidrográficas
}

Comparative study between SRTM images and army letters to the study of altimetric plan in watershed

\author{
Carlos Alberto Löbler'; Anderson Augusto Volpato Ssccoti'2; Lueni Gonçalves Terra ${ }^{3}$; \\ Tiago Ertel ${ }^{4}$; José Luiz Silvério da Silva ${ }^{5}$
}

'Geógrafo pela UFSM e Mestrando em Engenharia Ambiental pela UFSM.

${ }^{2}$ Geógrafo pela UFSM e Mestrando em Geografia pela Universidade Federal de Santa Maria.

${ }^{3}$ Geógrafa pela UFSM e Mestranda em Engenharia Civil pela UFSM.

${ }^{4}$ Acadêmico do curso de Engenharia Civil da UFSM.

${ }^{5}$ Professor Associado IV UFSM, Departamento de Geociências.

\section{Resumo}

O mapeamento do território brasileiro ainda não possui total cobertura. Uma forma de equacionar esta lacuna é através do uso de imagens de satélites. Um destes produtos são as imagens SRTM (Shuttle Radar Topography Mission), as quais possuem informações importantíssimas para estudos ambientais de escoamento superficial e fluxo subterrâneo. Ainda se carece de embasamento teórico quanto à confiabilidade desta base cartográfica. Nesse sentido, este trabalho objetiva comparar as informações referentes à altimetria presente nas imagens SRTM com informações contidas nas cartas topográficas. Essas foram confeccionadas pelo exército na década de 70, aplicados na bacia hidrográfica do Arroio do Veado, localizada entre os municípios de Santa Maria e Silveira Martins, na zona de transição entre a Depressão Central e o Planalto da Serra Geral, no Estado do Rio Grande do Sul, Brasil. Utilizou-se o programa ArcGIS extraindo-se as curvas de nível, as quais serviram de base, gerando-se o modelo numérico do terreno/MNT e mapa de declividade da bacia. Observou-se que os dados resultantes nas duas bases cartográficas são muitos semelhantes na escala 1:50.000 e bacia hidrográfica até 4.000 hectares. Conclui-se que nessas condições podem-se utilizar imagens SRTM, uma vez que apresentam facilidade de manuseio e ampla cobertura no país.

Palavras-chave: Mapeamento; base cartográfica; bacia hidrográfica

\begin{abstract}
The mapping of the Brazilian territory does not have full coverage. One way to solve this gap is through the use of satellite images. One of these products are the images SRTM (Shuttle Radar Topography Mission), which have very important information for environmental studies. Yet still lacks theoretical foundation regarding the reliability of this basemap. Thus, this study aims to compare the information on SRTM altimetry in the images with the information contained in topographic maps made by the brazilian army in the 70s. Was studied the watershed Arroio do Veado located between the cities of Santa Maria and Silveira Martins, in the transition zone between Central Depression and Serra Geral Plateau, in the Rio Grande do Sul state, southern Brazil. Using the ArcGIS software were extracted contour lines that served as bases and then generated the digital terrain model and slope map of the basin. It was observed that the data resulting from the two cartographic databases are very similar using a map scale 1: 50000 applied to watersheds with one area circa the 4,000 hectares. Was concluded, with these conditions may be used SRTM images, useful, handling and wide coverage in Brazil.
\end{abstract}

Keywords: mapping; cartographic base; watershed. 


\section{INTRODUÇÃO}

O mapeamento do território brasileiro foi iniciado pelo exército nos anos 60 . Sendo atualmente responsabilidade do Instituto Brasileiro de Geografia e Estatística (IBGE) e da Diretoria do Serviço Geográfico (DSG), contudo devido a extensão territorial do país e a diferentes problemas, como o alto custo destes mapeamentos, ainda existem áreas com vazios de informações e mapeamento em escala adequada para estudos ambientais, por exemplo. Sendo que apenas os mapeamentos de escala 1:1.000.000 conseguiram atingir $100 \%$ do território brasileiro.

O surgimento da cartografia por imagens de satélites pode, de certa forma, sanar esses vazios, já que as imagens como SRTM (Shuttle Radar Topography Mission) suprem algumas informações contidas nas cartas topográficas confeccionadas pelo exército brasileiro. O Projeto uso de satélites SRTM foi criado com o objetivo de obter informações altimétricas da superfície terrestre gerando uma base para estudos em diferentes áreas de pesquisa, como: Hidrologia, Geologia, Geomorfologia e muitas outras que possuem enfoque no meio ambiente e procuram descrever a superfície terrestre.

Os dados de elevação SRTM são resultado da missão realizada em conjunto pela NASA (National Aeronautics and Space Administration) e NIMA (National Imaging and Mapping Agency), dos EUA, com participação das agências espaciais da Alemanha (DLR) e da Itália (ASI). Ao longo de 11 dias, utilizando a técnica de interferometria de uma passagem, com duas antenas receptoras do SAR presas a uma haste mecânica, foram recobertos $80 \%$ da superfície terrestre (entre os paralelos $60^{\circ} \mathrm{N}$ e $56^{\circ} \mathrm{S}$ ), o que resultou em modelos tridimensionais com amplitude da grade de 90 metros (SRTM 3) são projetados para uma acurácia vertical e horizontal absoluta de aproximadamente 16 e 20 metros, respectivamente, com $90 \%$ de confiança (MEDEIROS et al., 2009).

Desde 1970, as metodologias empregadas em estudos ambientais sofreram grande impacto das novas geotecnologias, com a maior agilidade, objetividade, consistência e precisão na obtenção de bases de dados para fins de tomada de decisões geoespaciais (CARVALHO; BAYER, 2008). As imagens de satélites como as do SRTM necessitam processamento num ambiente SIG (Sistema de Informação Geográfica). Com isso a evolução dos SIGs também é importante para o melhor aproveitamento das imagens de satélites.
Muitos trabalhos tem sido realizados, em diferentes áreas das geociências, em diferentes escalas, para a verificação de bases cartográficas. Oliveira e Lopes (2007) concluíram que as imagens SRTM não foram confiáveis como base cartográfica. Nogueira et al. (2008) e Lemos et al. (SD) consideraram satisfatórios os resultados obtidos com o uso de imagens. Descovi Filho et al. (2008) realizaram estudos prévios sobre a temática, apontando também os erros de interpoladores matemáticos em bases cartográficas.

Nesse sentido e diante da necessidade de constatação de credibilidade de bases cartográficas, este trabalho tem como objetivo comparar a carta topográfica produzida pelo exército e as imagens de satélite SRTM. O processo de avaliação realizado comparou as curvas de nível geradas pelo SRTM, com as curvas nível existentes nas cartas topográficas, com uso de ferramentas de SIG, gerando-se dados altimétricos para obtenção de modelo numérico do terreno (mapa hipsométrico). Também com essas curvas foi possível gerar mapas de declividade. Assim obtendo informações sobre a confiabilidade do uso de dados extraídos de imagens SRTM.

\section{METODOLOGIA}

\section{Caracterização da Área de Estudo}

A bacia do Arroio do Veado está localizada entre os municípios de Santa Maria e Silveira Martins no interior do Estado do Rio Grande do Sul, conforme se observa na figura 1 , possui uma área de 4.016 ha e é um afluente da margem esquerda do Arroio Grande.

As bases cartográficas utilizadas foram adquiridas em diferentes fontes: a imagem SRTM foi obtida junto ao sítio da EMBRAPA (Empresa Brasileira de Pesquisa Agropecuária), que através do projeto monitoramento por satélite, disponibiliza imagens SRTM para pesquisadores. A partir destas imagens foram retiradas informações como: curvas de nível, com equidistâncias de 20 metros. Nessas imagens os pixels assumem valores de altitudes, que são estimados a partir do tempo em que a onda emitida pelo sensor remoto atinge a superfície terrestre e retorna ao satélite.

Na carta topográfica, em escala 1:50.000, após digitalizada e georreferenciada no ambiente de um programa, foi possível extrair informações como: hidrografia, curvas de nível de 20 em 20 metros e a delimitação da bacia.

O programa utilizado para interpolação 


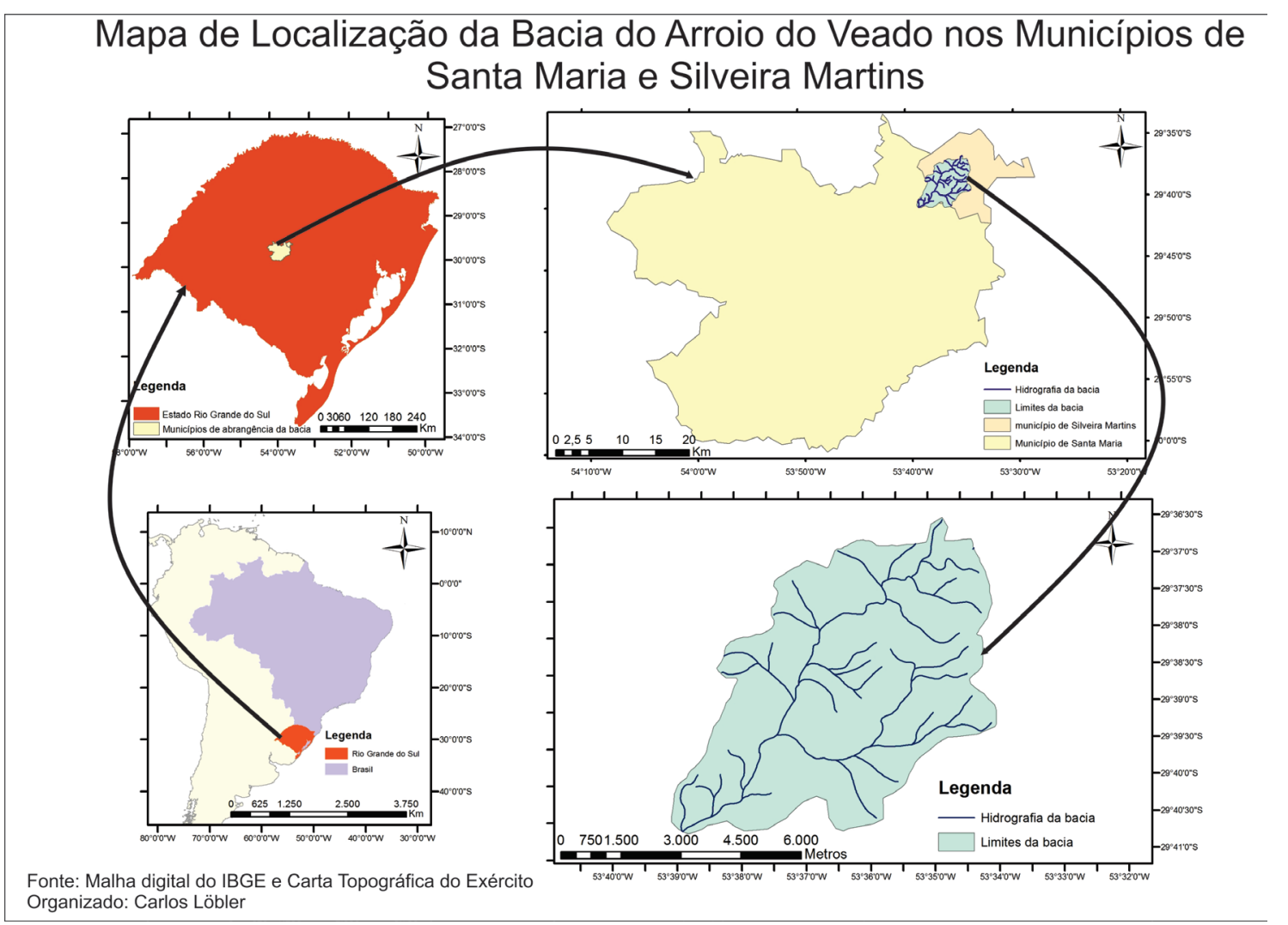

Figura 1 - Mapa de localização da área de estudo na porção central do RS.

dos dados e confecção dos mapas temáticos foi o ArcGis versão 10, produzido pela ESRI (Environmental Systems Research Institute). Foram criados diferentes mapas, relacionados ao modelo numérico do terreno, para comparação entre as bases cartográficas: dois altimétricos ou hipsométricos e ainda duas representações das declividades do terreno. Para uma análise minuciosa dos resultados encontrados foi realizada uma quantificação das classes utilizadas para o modelo numérico do terreno, a informação da área de cada classe foi obtida através da ferramenta ArcGis "xtools pro".

As classes utilizadas no mapa hipsométrico foram: <120m; 120-240m; 240-360m; 360-480m; $>480$. Essas classes foram definidas através da expressividade que cada uma representa, ou seja, foi a melhor forma de representar tematicamente as altitudes da bacia hidrográfica.

As classes usadas para representar a declividade foram: $<5 \% ; 5-10 \% ; 10-15 \% ; 15-30 \% ;>30 \%$. Pois essas representam as diferentes energias de deposição e erosão observadas no terreno.

Para gerar o mapa hipsométrico foi utilizada a ferramenta do arctoolsbox do ArcGis10 "topo to raster". Para gerar o mapa de declividade foi utilizada a ferramenta "Slope".

Para todos os mapas foi usado o datum
Córrego Alegre na zona 22, que tem como datum vertical Torres, RS

\section{RESULTADOS E DISCUSSÕES}

Com base nas curvas extraídas da carta topográfica do exército e da imagem SRTM foi possível espacializar a altitude da bacia do Arroio do Veado por meio de um modelo numérico do terreno (MNT). Com a atribuição das mesmas classes de altitude e mesmas cores para as classes nas duas bases cartográficas. Como pode ser observado nas figuras 2 e 3 , os mapas apresentaram diferenças insignificantes de informação altimétricas, mesmo sendo elas advindas de bases diferentes. Está pequena diferença não pode ser atribuída ao datum de referência uma vez que para todos os mapas foram usados o mesmo datum (Córrego Alegre zona 22) e o datum vertical Torres, RS

Através de cálculos de áreas realizados no programa ArcGIS 10, apontados na tabela 1, podemos confirmar as pequenas diferenças de resultados que se obtêm quando se trabalha com altitude usando uma carta topográfica do exército e as imagens SRTM. Nota-se que a diferença maior ocorreu na classe entre 120 e 240 metros, com cerca de 


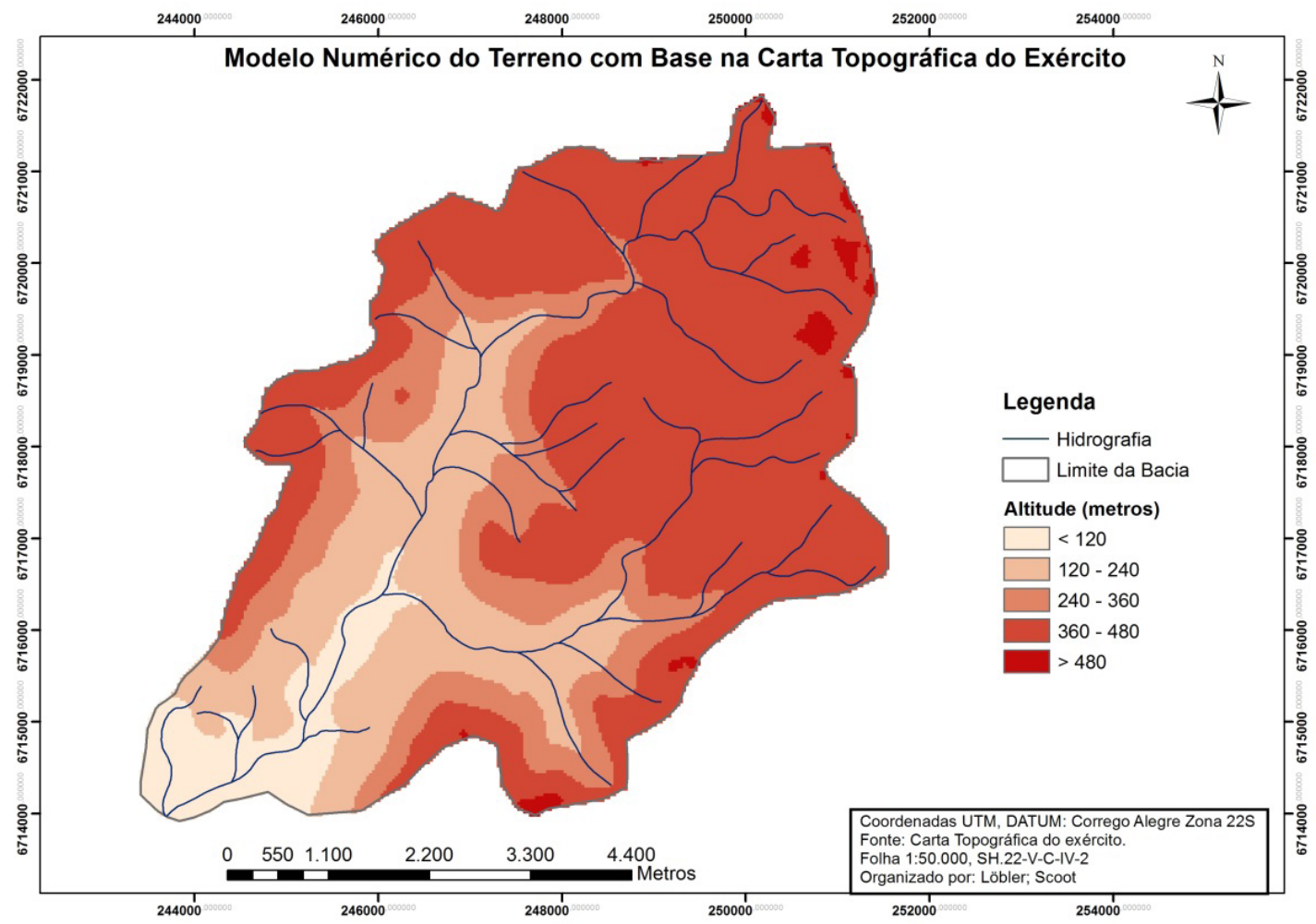

Figura 2 - Modelo Numérico do Terreno/MNT a partir da carta topográfica do exército de 1976.

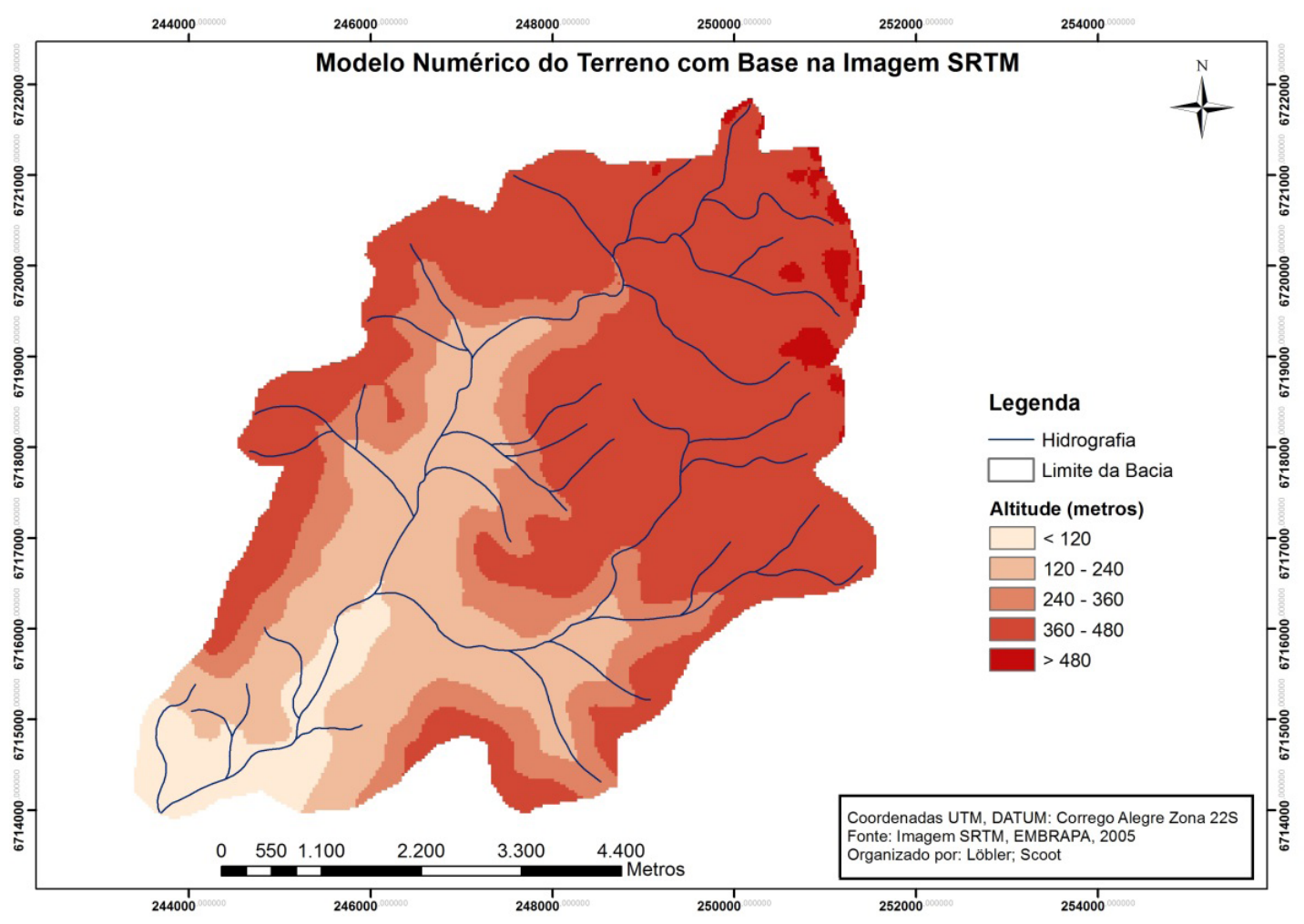

Figura 3 - Modelo Numérico do Terreno a partir de dados SRTM. 
Tabela 1 - Área correspondente a cada classe de altitude nas duas diferentes bases cartográficas.

\begin{tabular}{|l|c|c|c|}
\hline Classes & Área Carta (ha) & Área SRTM (ha) & Diferença(Carta - SRTM) (ha) \\
\hline$<120$ & 281,082735 & 254,718698 & 26,364037 \\
\hline $120-240$ & 814,979542 & 881,94219 & $-66,962648$ \\
\hline $240-360$ & 682,65814 & 660,203599 & 22,454541 \\
\hline $360-480$ & 2181,899716 & 2158,041767 & 23,857949 \\
\hline$>480$ & 56,344139 & 62,050717 & $-5,706578$ \\
\hline Total & 4016,964272 & 4016,956971 & 0,007301 \\
\hline
\end{tabular}

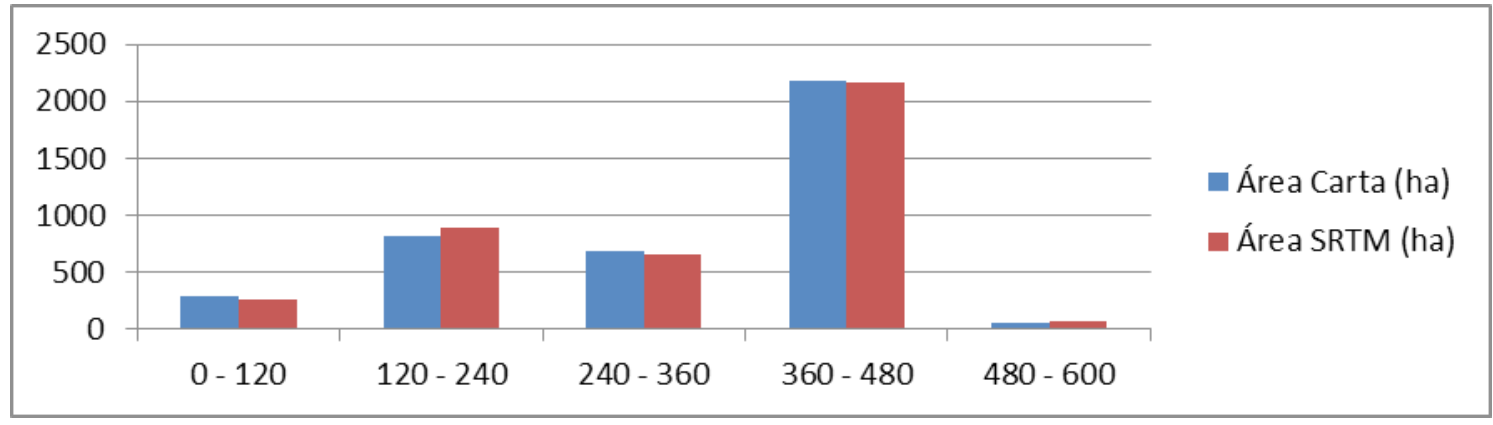

Figura 4 - Gráfico comparativo entre as classes de altitudes encontradas nas duas bases cartográficas

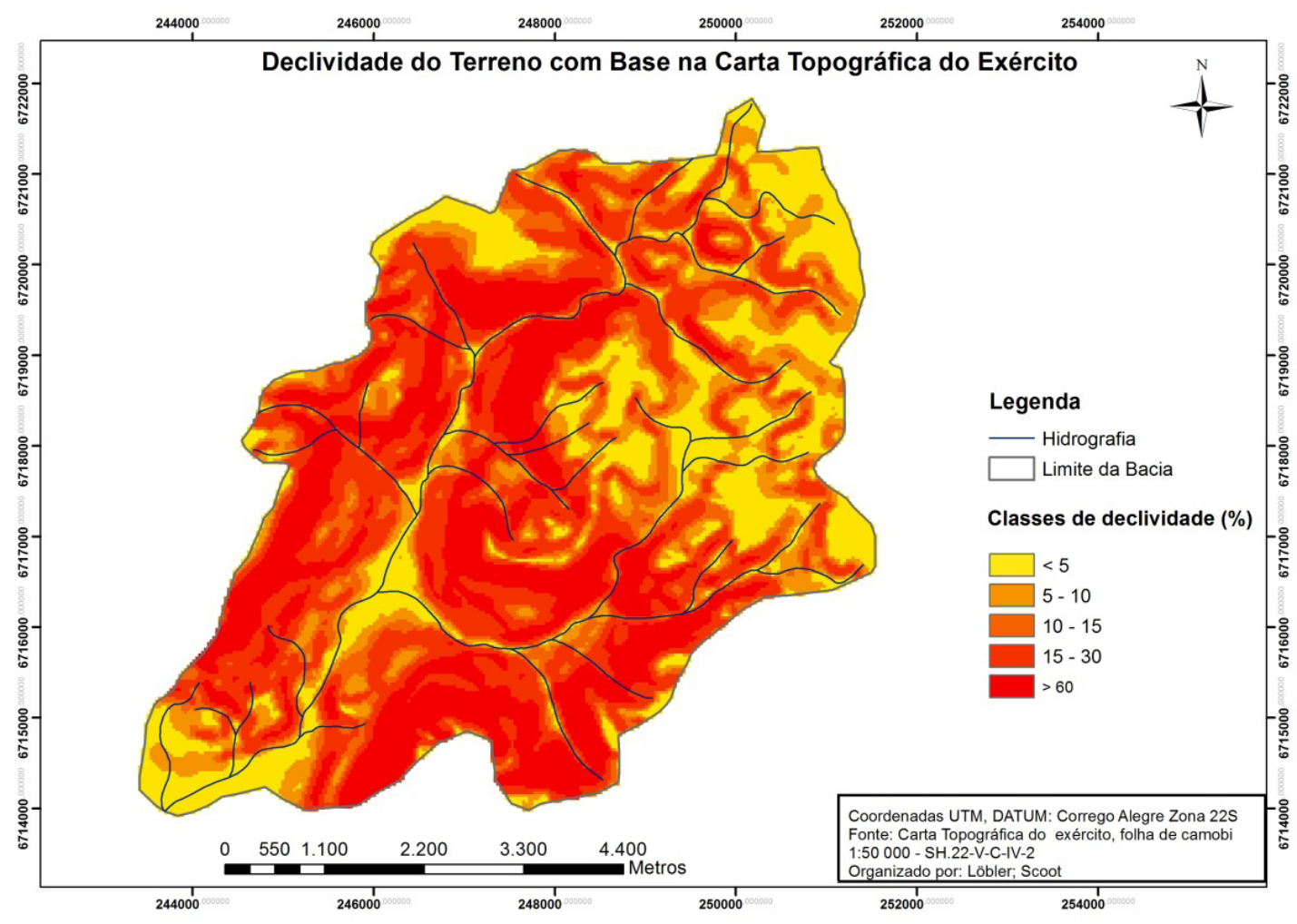

66 ha,

Figura 5 - Declividade da bacia com base na carta topográfica do exército de 1976. 


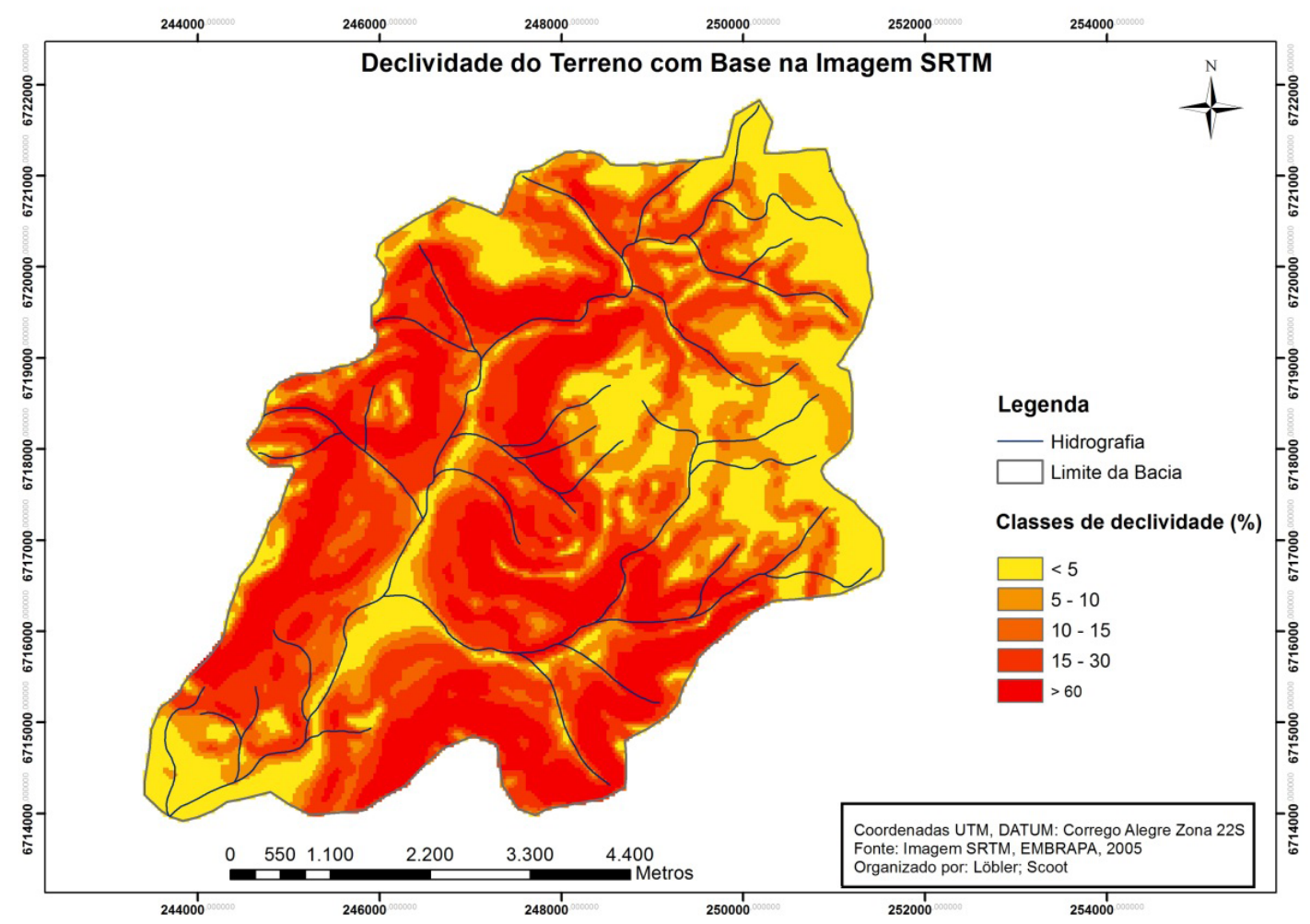

Figura 6 - Declividade da bacia com base na imagem SRTM.

entre as duas bases de informação.

$\mathrm{Na}$ figura 4 pode ser notada detalhadamente a diferença obtida com a manipulação dos dados. Observa-se que as curvas de nível retiradas da base carta topográfica do exército representada por classes altimétricas do modelo numérico do terreno apresentaram área maior nas classes, as quais estão representadas nas faixas: $<120 \mathrm{~m}, 240$ a $360 \mathrm{~m}$ e 360 a $480 \mathrm{~m}$. Enquanto que as classes da base cartográfica da imagem SRTM indicaram área maior nas classes: 120 a $240 \mathrm{~m}$ e $>480 \mathrm{~m}$.

As declividades geradas com as curvas extraídas nas duas bases cartográficas mostram resultados muito semelhantes. Notam-se apenas pequenas mudanças entre uma base e outra. Admitindo-se que a declividade é calculada pela distância entre as curvas de nível. Logo, pode-se dizer que o uso de imagens SRTM é confiável para estudos que envolvam declividade em bacias relativamente grandes e em escala que sejam superiores a 1: 50.000 .

Os mapas das figuras 5 e 6 mostram os resultados obtidos através dos dados extraídos das duas bases cartográficas.

\section{CONCLUSÕES}

O processamento dos dados permitiu inferir que, para estudos de altitude e declividade do terreno, a utilização das imagens SRTM apresentaram resultados satisfatórios, principalmente na escala 1:50.000. Então, podem servir de instrumento em estudos nas áreas em que há vazio de informações.

Contudo, devem ser consideradas em pesquisas usando imagens SRTM, as dimensões da bacia hidrográfica a ser avaliada. Uma vez que as imagens apresentam resolução por pixel de $90 \mathrm{~m}$, ou seja, cada pixel abrange uma área de $8.100 \mathrm{~m}^{2}$. Sendo assim nesses $8.100 \mathrm{~m}^{2}$ se terá o mesmo valor de altitude. Essa generalização pode não representar bem a realidade do terreno.

Além disso, o que pode influenciar também na escolha das imagens SRTM é o nível de exatidão pretendido com o trabalho. Recomenda-se o uso para estudos mais generalizados de bacias hidrográficas, também em análises de escoamento superficial e de tendência de fluxo subterrâneo. Outras formas de utilização das imagens necessitam de estudos mais aprofundados sobre a temática.

\section{AGRADECIMENTOS}

FAPERGS/CAPES, PIBIC/CNPq pelo apoio financeiro. 


\section{REFERÊNCIAS BIBLIOGRÁFICAS}

BRASIL. Exército Brasileiro. Diretoria de Serviço Geográfico - DSG, 1976. Carta Topográfica de Camobi. Folha SH.22-V-C-IV/2. Escala 1:50.000.

CARVALHO, T. M. de; BAYER, M. Utilização dos produtos da "shuttle radar topography mission" (SRTM) no mapeamento geomorfológico do estado de Goiás. Revista Brasileira de Geomorfologia. São Paulo - SP, n. 1, p. 35-41, 2008.

DESCOVI FILHO, L. V; CRUZ, R. C; CRUZ, J.C; SILVÉRIO DA SILVA, J. L.da Silva. Comparação entre produtos dos programas Spring 4.2 e Idrisi Kilimanjaro. Geografia. Ensino \& Pesquisa, v. 12, p. 4500-4514, 2008.

LEMOS, M. C. de; SOUZA, S. F. de; ROCHA, R. dos S. da. Avaliação da Qualidade dos Dados Altimétricos Derivado do Shuttle Radar Topographic Mission (SRTM): Resultados Preliminares. Disponível em: <http://www.ufpe.br/cgtg/ISIMGEO/CD/html/Fotogrametria $\% 20 \mathrm{e} \% 20$ Sensoriamento\%20Remoto/Artigos/f008.pdf>. Acesso em: 07 jun.2013.

MEDEIROS, L. C. FERREIRA, N. C. FERREIRA, L. G. Avaliação de Modelos Digitais de Elevação para Delimitação Automática de Bacias Hidrográficas. Revista Brasileira de Cartografia. Rio de Janeiro - RJ. n. 61/02, p. 138-151, 2009.

MIRANDA, E. E. de; (Coord.).Brasil em Relevo. Campinas: Embrapa Monitoramento por Satélite, 2005. Disponível em: <http://www.relevobr.cnpm. embrapa.br>. Acesso em: 22 mai. 2013.

OLIVEIRA, M. R. de; LOPES, E. E. Comparação entre os modelos de elevação gerados com dados SRTM e cartas do mapeamento sistemático nacional na escala de 1:50.000. In: SIMPÓSIO BRASILEIRO DE SENSORIAMENTO REMOTO, 12., 2007, Florianópolis. Anais eletrônicos... Florianópolis; INPE, 2007. 1 CD-ROM.

NOGUEIRA, T; JUNIOR, J . F. P; RODRIGUES, S. C. Compartimentação Morfológica com Base em Dados SRTM: Estudo de Caso Bacia do Rio Uberabinha, Uberlândia-MG. Rev. Geogr. Acadêmica. Goiânia - GO v.2, n. 2, p. 154-169, 2008. 